\title{
Shared Vulnerability of Two Synaptically-Connected Medial Temporal Lobe Areas to Age and Cognitive Decline: A Seven Tesla Magnetic Resonance Imaging Study
}

\author{
Geoffrey A. Kerchner, ${ }^{1}$ Jeffrey D. Bernstein, ${ }^{1}$ Michelle C. Fenesy, ${ }^{1}$ Gayle K. Deutsch, ${ }^{1}$ Manojkumar Saranathan, ${ }^{2}$ \\ Michael M. Zeineh, ${ }^{2}$ and Brian K. Rutt ${ }^{2}$ \\ ${ }^{1}$ Stanford Center for Memory Disorders, Department of Neurology and Neurological Sciences and ${ }^{2}$ Department of Radiology, Stanford University School of \\ Medicine, Stanford, California 94110
}

\begin{abstract}
The medial temporal lobe (MTL) is the first brain area to succumb to neurofibrillary tau pathology in Alzheimer's disease (AD). Postmortem human tissue evaluation suggests that this pathology propagates in an ordered manner, with the entorhinal cortex (ERC) and then CA1 stratum radiatum and stratum lacunosum-moleculare (CA1-SRLM) - two monosynaptically connected structures-exhibiting selective damage. Here, we hypothesized that, if ERC and CA1-SRLM share an early vulnerability to AD pathology, then atrophy should occur in a proportional manner between the two structures. We tested this hypothesis in living humans, using ultra-high field 7.0 T MRI to make fine measurements of MTL microstructure. Among a pool of age-matched healthy controls and patients with amnestic mild cognitive impairment and mild AD, we found a significant correlation between ERC and CA1-SRLM size that could not be explained by global atrophy affecting the MTL. Of the various structures that contribute axons or dendrites into the CA1-SRLM neuropil, only ERC emerged as a significant predictor of CA1-SRLM size in a linear regression analysis. In contrast, other synaptically connected elements of the MTL did not exhibit size correlations. CA1-SRLM and ERC structural covariance was significant for older controls and not patients, whereas the opposite pattern emerged for a correlation between CA1-SRLM and episodic memory performance. Interestingly, CA1SRLM and ERC were the only MTL structures to atrophy in older controls relative to a younger comparison group. Together, these findings suggest that ERC and CA1-SRLM share vulnerability to both age and AD-associated atrophy.
\end{abstract}

\section{Introduction}

In Alzheimer's disease (AD), neurofibrillary tau pathology appears first in the medial temporal lobe (MTL) (Braak et al., 2006). Dysfunction of this critical brain region leads to amnesia and heralds a progressive decline to dementia. With a desire to intervene as early as possible in this pathological cascade, there is mounting attention to the details of $\mathrm{AD}$ associated MTL degeneration.

Postmortem studies suggest a hierarchy of vulnerability to tau pathology in the MTL. Tangles appear in isolation in the perirhinal and entorhinal cortex (ERC) in what are presumed to be preclinical cases of AD (Braak et al., 2006). The hippocampal CA1 subfield is the next area affected, in which the burden falls hardest on the apical neuropil layer or the stratum radiatum and stratum

\footnotetext{
Received May 7, 2013; revised Aug. 4, 2013; accepted Sept. 12, 2013.

Author contributions: G.A.K., M.S., M.M.Z., and B.K.R. designed research; G.A.K., J.D.B., M.C.F., and G.K.D. performed research; G.A.K., J.D.B., M.C.F., and G.K.D. analyzed data; G.A.K. wrote the paper.

This work was supported by Alzheimer's Association Grant NIRG-11-205493, National Institutes of Health Grant K23AG042858, and the American Federation for Aging Research. We thank Christina Wyss-Coray and Michelle Fenesy for assistance in subject assessment

The authors declare no competing financial interests.

Correspondence should be addressed to Dr. Geoffrey A. Kerchner, Department of Neurology and Neurological Sciences, Stanford University School of Medicine, 300 Pasteur Drive, Room A343, MC5235, Stanford, CA 94305-5235. E-mail: kerchner@stanford.edu.

DOI:10.1523/JNEUROSCI.1915-13.2013

Copyright $\odot 2013$ the authors $\quad 0270-6474 / 13 / 3316666-07 \$ 15.00 / 0$
}

lacunosum-moleculare (CA1-SRLM; Fig. 1; Brady and Mufson, 1991; Braak and Braak, 1997a; Thal et al., 2000). Degeneration of the CA1-SRLM correlates tightly with memory loss and other signs of progressive dementia (Scheff et al., 2007; Kerchner et al., 2012).

Notably, CA1-SRLM is a synaptic target of ERC neurons (Fig. 1) (Insausti and Amaral, 2012). In mouse models of AD, tau pathology appears to propagate transynaptically from ERC neurons to their targets (de Calignon et al., 2012; Harris et al., 2012; Liu et al., 2012), perhaps in a prion-like manner (Frost et al., 2009). These observations suggest one possible explanation for the hierarchical vulnerability observed in humans. A broader notion has emerged, not only in AD but also in other neurodegenerative diseases, that neural dysfunction and atrophy extend in a network-dependent manner around the brain rather than contiguously (Seeley et al., 2009).

In living humans, MTL structure may be studied using magnetic resonance imaging (MRI), which reveals ERC and CA1 atrophy among patients with $\mathrm{AD}$ or amnestic mild cognitive impairment (aMCI), a clinical precursor to $\mathrm{AD}$ (Xu et al., 2000; Killiany et al., 2002; Adachi et al., 2003; Csernansky et al., 2005; Apostolova et al., 2006; Mueller et al., 2007, 2010). We reported previously that the fine-layered anatomy of the hippocampus is visible using ultra-high field 7 T MRI (Kerchner, 2011) and that thinning of the CA1-SRLM differentiates patients with $\mathrm{AD}$ from 


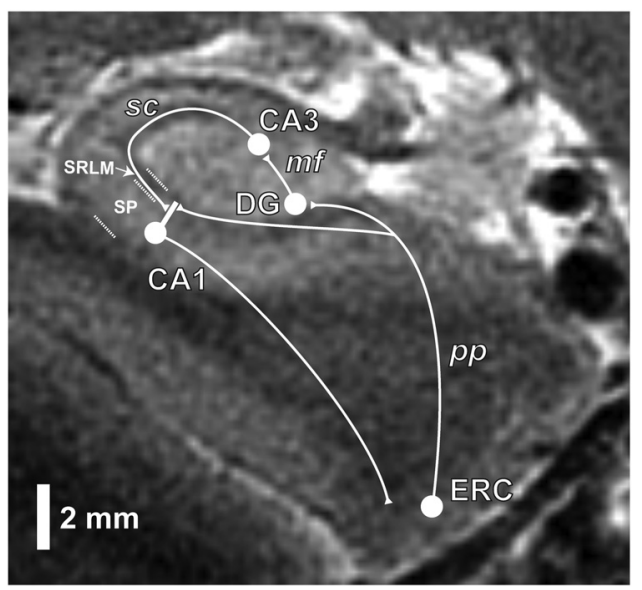

Figure 1. MTL connections. Overlaid onto an image of the left hippocampus from a healthy $O C$ subject in this study is a schematic of the major pathways within the MTL. Neurons in layers II/III of the ERC project, via the perforant pathway (pp), to granule cells in the DG and to CA1 pyramidal neurons. Granule cell axons, or mossy fibers ( $\mathrm{mf}$ ), terminate onto CA3 pyramidal neurons, which supply recurrent axons back to CA3 (not labeled) and project via Schaffer collateral axons (sc) to CA1 pyramidal neurons. The CA1 axons project to the subiculum (not labeled) and back to deep layers of the ERC. Dashed lines delineate the strata of CA1, including the SP and SRLM.

age-matched healthy controls (Kerchner et al., 2010) and correlates with memory performance (Kerchner et al., 2012).

High-resolution imaging offers an opportunity to test, in vivo, hypotheses derived from animal models and postmortem human tissue research. Here, we hypothesized that, if pathology (and, in turn atrophy) indeed arises earliest in the ERC and CA1-SRLM, then there should be a structural correlation between these two areas. To test this hypothesis, we studied age-matched older adults across a cognitive spectrum, including healthy controls and patients with aMCI and mild AD, using 7 T MRI. To investigate the effect of age on the relationship between ERC and CA1SRLM structure, we also included a group of young healthy controls.

\section{Materials and Methods}

Subjects. Each subject provided written, informed consent in accordance with a protocol approved by the Stanford Institutional Review Board. Patients with aMCI and mild AD were recruited from the Stanford Center for Memory Disorders. Inclusion criteria included sufficient English language skills to participate in the neuropsychological assessment and (1) a diagnosis of probable $\mathrm{AD}$ (amnestic presentation) according to the National Institute on Aging-Alzheimer's Association criteria (McKhann et al., 2011) and a Clinical Dementia Rating (CDR) score of 0.5 or 1 (Morris, 1993), or (2) a diagnosis of MCI according to the National Institute on Aging-Alzheimer's Association criteria (Albert et al., 2011), an objective memory impairment, and a $\mathrm{CDR}<1$. We also recruited healthy older control (OC) subjects from the community, selected to approximate the ages of the patient cohorts. Inclusion criteria included no cognitive complaint, no neuropsychological abnormality, a normal neurological examination, and $\mathrm{CDR}=0$. Exclusion criteria for all participants included any contraindication to MRI, a history of stroke or other structural brain abnormality, or the presence of any neurological or medical condition other than AD that could interfere with normal cognition. The healthy younger control (YC) subjects, recruited from the community, underwent imaging but not clinical assessment. Both sexes were eligible for this study, and the characteristics of all subjects appear in Table 1.

Clinical assessment. Each older subject underwent a clinical evaluation that included a history, physical examination, and neurological examination by a physician at the Stanford Center for Memory Disorders. With an identified study partner (usually a spouse), each older subject underwent a functional assessment that included the CDR (Morris, 1993), Neuropsychiatric Inventory (Cummings et al., 1994), the Geriatric Depression Scale (Yesavage, 1988), and the Functional Assessment Questionnaire (Pfeffer et al., 1982). A trained psychometrician administered a standardized neuropsychological battery covering episodic memory, language, visuospatial function, working memory, and executive function, as described previously (Kerchner et al., 2012). Episodic memory tests include the Hopkins Verbal Learning Test-Revised, the Brief Visuospatial Learning Test-Revised, and the Logical Memory subtest of the Wechsler Memory Scale, Third Edition, from which we derived composite metrics of immediate free recall memory, delayed free recall memory, and delayed recognition memory, as described previously (Kerchner et al., 2012). The faculty of the Center, including neurologists and neuropsychologists, discussed each potential subject in a consensus conference to determine a research diagnosis. For each subject, all study metrics, including imaging, were acquired within an average window of $48 \mathrm{~d}$ (range of 1-106).

Image acquisition. Subjects were scanned on a 7 T GE Signa HDx whole-body MRI scanner (GE Healthcare), as described previously (Kerchner et al., 2012), using a 32-channel radiofrequency receive head coil contained within a quadrature transmit coil (Nova Medical). The subject's head was stabilized by packing foam between the temples and the inner surface of the receive coil to minimize motion during the scan, and a plethysmograph was placed on a finger on the right hand to monitor peripheral pulse. Imaging was targeted to the MTL, with acquisition of oblique coronal images oriented perpendicularly to the longitudinal axis of the hippocampus, using a T2-weighted fast spin echo sequence: effective echo time, $49 \mathrm{~ms}$; repetition time (TR), 5-6 s (cardiac gated, with the R-R interval set for each subject according to their average heart rate so as to achieve a TR within this range); nominal excitation flip angle, $90^{\circ}$; tailored refocusing flip angle from $160^{\circ}$ to $130^{\circ}$; echo train length, 8 ; bandwidth, $20.8 \mathrm{kHz}$; number of excitations, 1 ; slice thickness, $1.5 \mathrm{~mm}$; slice gap, $0.5 \mathrm{~mm}$; field of view, $170 \times 170 \mathrm{~mm}^{2}$; acquisition matrix, $768 \times 768$, yielding acquired voxels measuring $0.22 \times 0.22 \times 1.5 \mathrm{~mm}^{3}$. Images were interpolated on the scanner by zero filling $k$-space to a $1024 \times 1024$ matrix yielding a reconstructed voxel size of $0.166 \times$ $0.166 \times 1.5 \mathrm{~mm}^{3}$. Specific absorption rate was $\sim 2.5 \mathrm{~W} / \mathrm{kg}$. Typically, $16-18$ slices were obtained per subject covering most or all of the head of the hippocampus, the entire body, and some of the tail. Scan time was typically just $<10 \mathrm{~min}$.

Image analyses. We visualized images with the OsiriX 3.9 software package (http://www.osirix-viewer.com; Rosset et al., 2004) and with FSL-View, a component of the FSL (for FMRIB Software Library) version 4.1 software package (http://www.fmrib.ox.ac.uk/fsl/). A single scientist (G.A.K.) selected slices and delineated of regions of interest. We described previously the landmarks used for subfield identification and the methods used to derive subfield metrics (Kerchner et al., 2012). In brief, we determined CA1-SRLM and CA1 stratum pyramidale (CA1-SP) widths using a semiautomated method and manually determined the width of the ERC and the cross-sectional areas of the entire hippocampus and the combined dentate gyus and CA3 subfields (DG/CA3; we combined these two areas because no physical boundary appears at this imaging resolution). For each subfield, we averaged raw metrics across slices to yield one value per side per subject and then averaged the two sides to yield a single subfield metric per subject. When areas and widths were compared in statistical analyses, we used the square root of the areas so that all metrics were one dimensional. Because of the large slice thickness, skip between slices, and incomplete coverage of the hippocampus, all necessary to achieve a very high in-plane resolution and signal-to-noise ratio, we constrain our analyses to one- or two-dimensional metrics rather than volumes; for example, we report average hippocampal crosssectional area rather than hippocampal volume.

Statistics. Results are indicated as mean \pm SD. We used SPSS version 21.0 for all statistical comparisons. The threshold for statistical significance was $p<0.05$ using two-tailed tests. 
Table 1. Participant characteristics

\begin{tabular}{|c|c|c|c|c|c|}
\hline & $Y C(n=9)$ & $O C(n=18)$ & $\mathrm{aMCl}(n=15)$ & $\mathrm{AD}(n=11)$ & $p$ values \\
\hline \multicolumn{6}{|l|}{ Demographics } \\
\hline Age (years) & $28.2 \pm 4.1$ & $70.2 \pm 6.2$ & $73.2 \pm 5.5$ & $69.5 \pm 9.3$ & 0.32 \\
\hline Females (\%) & $2(22 \%)$ & $10(56 \%)$ & $7(47 \%)$ & $6(55 \%)$ & 0.87 \\
\hline Education (years) & & $16.4 \pm 1.8$ & $16.9 \pm 2.5$ & $15.8 \pm 2.9$ & 0.50 \\
\hline \multicolumn{6}{|c|}{ Functional and global cognitive scales } \\
\hline MMSE & & $29.7 \pm 0.6$ & $27.5 \pm 2.7^{*}$ & $25.1 \pm 2.6^{* *}$ & $<0.001$ \\
\hline Full Scale IQ & & $120 \pm 10$ & $117 \pm 14$ & $95 \pm 16^{* *}$ & $<0.001$ \\
\hline CDR & & 0 & $0.5 \pm 0.1^{* *}$ & $0.8 \pm 0.3^{* *}$ & $<0.001$ \\
\hline CDR-SB & & 0 & $1.6 \pm 1.2^{* *}$ & $4.1 \pm 1.3^{* *}$ & $<0.001$ \\
\hline \multicolumn{6}{|c|}{ Episodic memory composite scores ${ }^{a}$} \\
\hline Immediate & & $0.73 \pm 0.13$ & $0.36 \pm 0.16^{* *}$ & $0.26 \pm 0.11^{* *}$ & $<0.001$ \\
\hline Delayed & & $0.76 \pm 0.13$ & $0.28 \pm 0.22^{* *}$ & $0.15 \pm 0.14^{* *}$ & $<0.001$ \\
\hline Recognition & & $0.76 \pm 0.07$ & $0.47 \pm 0.18^{* *}$ & $0.37 \pm 0.25^{* *}$ & $<0.001$ \\
\hline \multicolumn{6}{|c|}{ Other neuropsychological metrics ${ }^{b}$} \\
\hline Digit span & & $18.2 \pm 4.0$ & $15.3 \pm 3.2^{*}$ & $12.6 \pm 2.7^{* *}$ & $<0.001$ \\
\hline Category fluency & & $23.4 \pm 6.4$ & $15.4 \pm 5.0^{* *}$ & $11.3 \pm 4.5^{* *}$ & $<0.001$ \\
\hline Trails B & & $80 \pm 30$ & $110 \pm 63$ & $206 \pm 65^{* *}$ & $<0.001$ \\
\hline Boston naming test & & $28.8 \pm 1.2$ & $24.2 \pm 6.0^{* *}$ & $24.8 \pm 2.9^{*}$ & $<0.01$ \\
\hline Figure copy & & $18.6 \pm 1.7$ & $18.0 \pm 2.2$ & $12.1 \pm 7.0^{* *}$ & $<0.001$ \\
\hline Block design & & $37.7 \pm 11.2$ & $37.8 \pm 10.5$ & $14.9 \pm 12.8^{* *}$ & $<0.001$ \\
\hline Matrix reasoning & & $25.2 \pm 3.9$ & $23.7 \pm 4.1$ & $14.0 \pm 7.8^{* *}$ & $<0.001$ \\
\hline
\end{tabular}

Except as indicated, data are reported as mean \pm SD. Statistical comparisons were made only for the $O C$, aMCI, and AD participants, excluding YCs, with $p$ values in the rightmost column. One-way ANOVAs were performed for all comparisons, except gender proportions (Pearson's $\chi^{2}$ test). ${ }^{*} p<0.05$ or ${ }^{* *} p<0.01$ compared with OCs by Dunnett's two-sided post hoc analysis. CDR-SB, CDR sum of boxes; MMSE, mini-mental state examination.

${ }^{a}$ See Materials and Methods for derivations of immediate free recall ("immediate"), delayed free recall ("delayed"), and delayed recognition ("recognition") composite scores, which range from 0 to 1.

${ }^{b}$ Raw scores (for Trails B, higher scores are worse).

\section{Results}

OC subjects and patients with aMCI and AD did not differ significantly by age, gender, or education (Table 1).

We found a CA1-SRLM/ERC width ratio of $0.25 \pm 0.03$ across our subjects. Neither age nor diagnosis affected this ratio (Fig. 2A; Table 2), although the size of each area decreased as a function of age and cognitive impairment (Fig. $2 B, C$; Table 2). The ratio did not vary by gender ( $p=0.75$ by $t$ test). Ratios between other synaptically connected subfields, including CA1-SP to ERC, DG/CA3 to ERC, and DG/CA3 to CA1-SRLM, were higher in OCs than YCs, likely reflecting age-associated atrophy that affected ERC and CA1-SRLM but not DG/CA3 or CA1-SP (Table 2). These ratios appeared constant across the spectrum of cognitive decline among the older participants (Table 2).

Perhaps the stability of the CA1-SRLM-to-ERC width ratio is a nonspecific result of global, proportionate atrophy of the MTL. To test this possibility, we examined partial correlations between MTL subfield metrics among older adult subjects, controlling for hippocampal area, a proxy for hippocampal volume (see Materials and Methods) and a marker of the overall burden of atrophy. As illustrated in Table 3, significant correlations emerged between ERC and CA1-SRLM widths (Fig. 3) and between ERC and CA1-SP but not between other areas. Adding age as a covariate did not alter these partial correlations. The significant partial correlation between CA1-SRLM and ERC widths may have been driven by the OC group (Fig. 4): when each diagnostic group was analyzed alone, a significant partial correlation between these metrics emerged for OCs $(r=0.62, p<0.01)$ but not patients with aMCI $(r=0.30, p=0.29$; with the outlier described in Fig. 3 removed, $r=0.47, p=0.11)$ or $\mathrm{AD}(r=0.02, p=0.96)$. Unadjusted Pearson's correlations between CA1-SRLM and ERC widths were significant for OCs $(r=0.64, p<0.01)$, patients with mild AD $(r=0.61, p<0.05)$, and marginally for patients with aMCI $(r=0.46, p=0.088$; with the outlier described in Fig. 3 removed, $r=0.58, p<0.05)$. Among YCs, CA1-SRLM and ERC widths did not correlate significantly, whether corrected for hippocampal area $(r=-0.39, p=0.35)$ or not $(r=-0.39$, $p=0.30)$.

Notably, DG/CA3, which connects both with the ERC and CA1 (Fig. 1), did not exhibit significant hippocampal areaadjusted partial correlations with any other subfield metric (Table 3). Using Steiger's $Z$ test to compare correlation coefficients (Meng et al., 1992), the relationship between ERC and CA1SRLM illustrated in Figure 3 and Table 3 was significantly stronger than between ERC and DG/CA3 $(p<0.01)$; the same was true for the correlation between ERC and CA1-SP $(p<0.05)$.

We next performed a linear regression analysis as a more robust method of testing the strength of the relationship between CA1-SRLM and ERC. As illustrated in Figure 1, CA3 and ERC provide the important axonal projections to this neuropil layer, and dendrites therein belong mainly to CA1 pyramidal neurons, whose cell bodies lie in CA1-SP; thus DG/CA3, ERC, and CA1-SP could each influence the size of CA1-SRLM. We designed a hierarchical linear regression model with this anatomy in mind: with CA1-SRLM as the dependent variable, we combined older adult data across diagnostic groups and entered hippocampal area and diagnosis into the model first as nuisance variables and then entered ERC width, CA1-SP width, and DG/CA3 area. As illustrated in Table 4, we found that, although CA1-SRLM does change with hippocampal area, the addition of the other MTL anatomical metrics accounted for $14 \%$ more of the variance. Of those metrics, only ERC width was a significant predictor of CA1-SRLM width. To investigate whether CA1-SP width is influenced by its afferents, we performed a similar hierarchical regression analysis and found no significant association with DG/ CA3 area or ERC width $\left(R^{2}\right.$ change for Step 2 was $\left.0.05, p=0.19\right)$.

Finally, we examined correlations between hippocampal subfield metrics and memory performance. We reported previously that, among patients with mild $\mathrm{AD}$, hippocampal subfield metrics correlated with delayed memory composite scores (Kerchner et al., 2012). With a slightly larger sample size, we reproduced this 

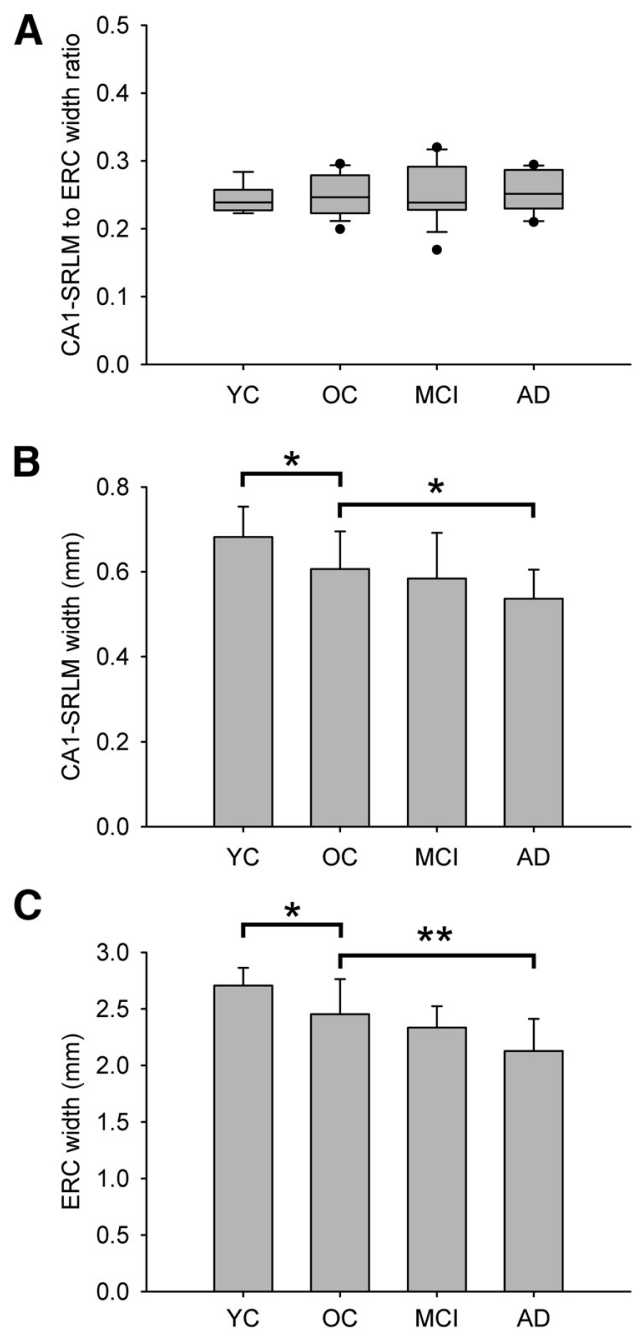

Figure 2. Effects of age and cognitive impairment on CA1-SRLM and ERC widths. The CA1SRLM-to-ERC width ratio $(\boldsymbol{A})$ did not differ across groups ( $p=0.97$ by one-way ANOVA). Box plots indicate the median (central line), 25th and 75th percentiles (lower and upper limits of boxes), 10th and 90th percentiles (lower and upper error bars), and outliers (dots). Both CA1SRLM width $(\boldsymbol{B})$ and ERC width $(\boldsymbol{C})$ decreased with age and with a diagnosis of AD. One-way ANOVAs were significant (CA1-SRLM, $\left.F_{(3,49)}=4.67, p<0.01 ; \operatorname{ERC}, F_{(3,49)}=9.22, p<0.001\right)$, and a priori contrasts of $Y C$ and $A D$ groups to $O C$ s yielded significant results $\left({ }^{*} p<0.05\right.$, ${ }^{* *} p<$ $0.01)$; a priori contrasts between aMCl and $0 \mathrm{Cs}$ were not significant. Error bars represent SDs. YC, $n=9 ; 0 \mathrm{C}, n=18 ; \mathrm{aMCl}, n=15 ; \mathrm{AD}, n=11$.

Table 2. Subfield metrics and ratios

\begin{tabular}{lllllr}
\hline \multicolumn{1}{l}{$Y C$} & \multicolumn{1}{l}{ OC } & \multicolumn{1}{c}{ aMCl } & \multicolumn{1}{l}{ AD } & $p$ values \\
\hline Raw metrics & & & & \\
ERC (mm) & $2.71 \pm 0.16^{*}$ & $2.45 \pm 0.31$ & $2.33 \pm 0.19$ & $2.13 \pm 0.28^{* *}$ & $<0.001$ \\
CA1-SRLM (mm) & $0.68 \pm 0.07^{*}$ & $0.61 \pm 0.09$ & $0.58 \pm 0.11$ & $0.54 \pm 0.07^{*}$ & 0.006 \\
CA1-SP (mm) & $1.55 \pm 0.23$ & $1.66 \pm 0.22$ & $1.57 \pm 0.19$ & $1.39 \pm 0.22^{*}$ & 0.018 \\
DG/CA3 (cm $\left.{ }^{a}\right)$ & $0.36 \pm 0.02$ & $0.37 \pm 0.04$ & $0.37 \pm 0.06$ & $0.33 \pm 0.03^{*}$ & 0.035 \\
Ratios $^{b}$ & & & & \\
CA1-SRLM to ERC & $0.25 \pm 0.04$ & $0.25 \pm 0.03$ & $0.25 \pm 0.04$ & $0.25 \pm 0.03$ & 0.97 \\
CA1-SP to ERC & $0.58 \pm 0.09^{* *}$ & $0.68 \pm 0.10$ & $0.68 \pm 0.10$ & $0.65 \pm 0.04$ & 0.03 \\
DG/CA3 to ERC & $0.13 \pm 0.01^{*}$ & $0.15 \pm 0.02$ & $0.16 \pm 0.02$ & $0.16 \pm 0.02$ & 0.025 \\
DG/CA3 to & $0.53 \pm 0.06^{*}$ & $0.62 \pm 0.10$ & $0.64 \pm 0.11$ & $0.62 \pm 0.09$ & 0.042 \\
CA1-SRLM & & & & & \\
\end{tabular}

Data are reported as mean \pm SD. One-way ANOVAs were performed for each metric and ratio, yielding the $p$ values in the rightmost column. A priori contrasts were performed comparing $\mathrm{YCs}$ and patients with $\mathrm{aMCl}$ or $\mathrm{AD}$ to $\mathrm{OCs}$. ${ }^{*} p<0.05 ;{ }^{* *} p<0.01$.

${ }^{a}$ As detailed in Material and Methods, the image analysis yielded DG/CA3 cross-sectional areas, but we use the square root of this metric to keep all metrics one dimensional.

${ }^{b}$ Ratio pairs were chosen on the basis of anatomical connections (Fig. 1).
Table 3. Intersubfield correlations among older adults

\begin{tabular}{lccc}
\hline & ERC & CA1-SRLM & CA1-SP \\
\hline CA1-SRLM & $0.41^{* *}$ & & \\
CA1-SP & $0.33^{*}$ & -0.20 & \\
DG/CA3 & -0.21 & -0.25 & -0.14 \\
\hline
\end{tabular}

Partial correlation coefficients are adjusted for hippocampal area. ${ }^{*} p<0.05 ;{ }^{* *} p<0.01$.

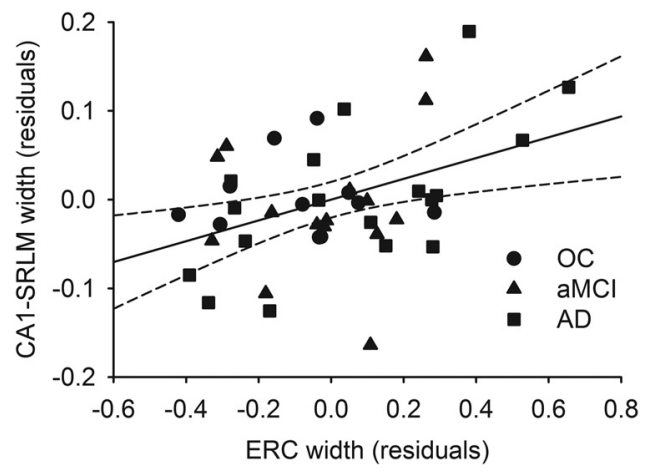

Figure 3. Correlation between CA1-SRLM width and ERC width. Residuals from a partial correlation between CA1-SRLM width and ERC width, controlling for hippocampal crosssectional area, are plotted with a regression line and $95 \%$ confidence interval for the older adults in this study $(O C, n=18 ; \mathrm{aMCl}, n=15 ; \mathrm{AD}, n=11)$. Both axes represent residual values after a regression with hippocampal area. Incidental note is made of an outlier, a patient with aMCI with the lowest CA1-SRLM width residual and the only older subject whose CA1-SRLMto-ERC width ratio was $>3$ median absolute deviations from the group median (the lowest point on Fig. 2A). Excluding this individual did not affect the statistics of this partial correlation or the linear regression analysis (Table 4).

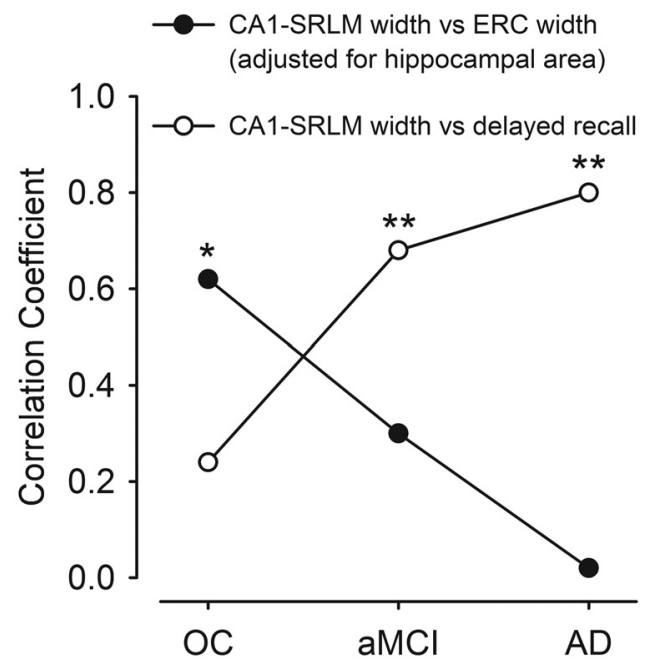

Figure 4. Contrasting structural and behavioral correlations across a cognitive spectrum. For the CA1-SRLM width to ERC width correlations, partial Pearson's correlation coefficients are illustrated after adjusting each metric for hippocampal cross-sectional area (as in Fig. 3 and Table 3). For the CA1-SRLM width to delayed recall composite score correlation, unadjusted Pearson's coefficients are illustrated (see Table 5). Significant coefficients are labeled ${ }^{*} p<$ $\left.0.05,{ }^{* *} p<0.01\right) .0 C, n=18 ; \mathrm{aMCl}, n=15 ; \mathrm{AD}, n=11$.

finding here (Fig. 4; Table 5). In addition, among patients with aMCI, CA1-SRLM width correlated significantly with delayed free recall performance. It might be expected that, because of the shared variance between CA1-SRLM and ERC widths, ERC width should also correlate with the same performance metric; although this correlation did not reach statistical significance, a trend emerged (Table 5). Removal of the aMCI outlier (Fig. 3 and see above) did not affect this pattern of significant correlations. 
Table 4. Predictors of CA1-SRLM width from linear regression

\begin{tabular}{lccllll}
\hline Variable & $B^{a}$ & $\mathrm{SEB}$ & $\beta^{b}$ & $\mathrm{R}^{c}$ & Adjusted $R^{2}$ & $R^{2}$ change \\
\hline Step 1 & & & & 0.64 & 0.37 & $0.41^{* * *}$ \\
$\quad$ Hippocampal size & 0.78 & 0.17 & $0.64^{* * *}$ & & & \\
AD $^{d}$ & 0 & 0.03 & 0 & & & \\
aMCl $^{d}$ & -0.01 & 0.03 & -0.04 & & & \\
Step 2 & & & & 0.74 & 0.47 & $0.14^{*}$ \\
Hippocampal size & 0.96 & 0.30 & $0.80^{* *}$ & & & \\
AD $^{d}$ & 0.02 & 0.03 & 0.08 & & & \\
aMCl $^{d}$ & 0 & 0.02 & 0.01 & & & \\
ERC & 0.13 & 0.05 & $0.40^{* *}$ & & & \\
CA1-SP & -0.07 & 0.06 & -0.18 & & & \\
DG/CA3 & -0.51 & 0.43 & -0.25 & & & \\
\hline
\end{tabular}

${ }^{*} p<0.05 ;{ }^{* *} p<0.01 ;{ }^{* * *} p<0.001$.

${ }^{a}$ Unstandardized coefficient.

${ }^{b}$ Standardized coefficient.

'Correlation coefficient.

${ }^{d}$ Categorical dummy variable indicating group membership.

Table 5. Structural correlates of episodic memory performance

\begin{tabular}{llllr}
\hline & ERC & CA1-SRLM & CA1-SP & DG/CA3 \\
\hline OC & & & & \\
Immediate & 0.29 & 0.30 & 0.06 & -0.06 \\
Delayed & 0.23 & 0.24 & 0.03 & 0.02 \\
$\begin{array}{l}\text { Recognition } \\
\text { aMCl }\end{array}$ & 0.23 & 0.37 & 0.15 & 0.10 \\
Immediate & 0.15 & 0.36 & & \\
Delayed & $0.50^{\wedge}$ & $0.68^{* *}$ & 0.08 & 0.15 \\
Recognition & $0.47^{\wedge}$ & 0.42 & 0.14 & 0.47 \\
AD & & & 0.02 & 0.34 \\
Immediate & $0.63^{*}$ & $0.57^{\wedge}$ & & 0.41 \\
Delayed & $0.72^{*}$ & $0.80^{* *}$ & $0.71^{*}$ & $0.07^{\wedge}$ \\
Recognition & $0.67^{*}$ & $0.70^{*}$ & 0.45 & 0.03 \\
\hline
\end{tabular}

Pearson's $r$ values are reported. See Materials and Methods for derivations of immediate free recall ("immediate"), delayed free recall ("delayed"), and delayed recognition ("recognition") composite scores. ${ }^{*} p<0.05$, ${ }^{* *} p<0.01$; ^nonsignificant trend, defined as $p<0.1$.

Among OCs, there was no correlation between any subfield metric and any memory composite score (Table 5).

\section{Discussion}

We found that ERC and CA1-SRLM exhibit structural covariance among older individuals. Consistent with the notion that these two synaptically connected areas of the MTL share an early vulnerability to degeneration in $\mathrm{AD}$, we found a significant correlation between their thicknesses among age-matched older adults across a cognitive spectrum. Among pooled older participants and for OCs alone, this correlation persisted after accounting for global hippocampal size. Although we are not the first to suggest that AD pathology selectively affects this ERC-CA1SRLM pathway (Braak and Braak, 1997a; Thal et al., 2000; Lace et al., 2009), we provide a new, in vivo perspective on this hypothesis.

The relationship between CA1 and ERC was unique, and we did not observe structural covariance between other MTL structures (Table 3). The DG is a major target of ERC neurons (Fig. 1), and yet DG/CA3 size did not correlate with ERC thickness. This is consistent with observations from postmortem tissue, in which tau pathology selectively affects the apical dendrites of CA1 neurons earlier or more robustly than the DG (Brady and Mufson, 1991; Braak and Braak, 1997a; Thal et al., 2000). Using lowerfield MRI, other groups have reported disproportionate atrophy of the CA1 subfield in the context of aMCI and AD (Adachi et al., 2003; Csernansky et al., 2005; Apostolova et al., 2006; Mueller et al., 2007, 2010), although one group described disproportionate involvement of the DG (Yassa et al., 2010).

Conversely, the absence of an observed relationship between DG/CA3 and other MTL structures may reflect limitations of our technique: by collapsing the DG and CA3 into a single measurable subfield (because no border is discernible even at this high resolution), we may miss focal structural changes isolated to one or the other structure. In addition, the hypointense band separating CA1-SP from DG/CA3 on our images (Fig. 1) may contain a portion of the outer molecular layer of the DG, a neuropil area that contains the apical neurites of DG granule cells and perforant pathway axons and is susceptible to tau pathology in the course of AD (Brady and Mufson, 1991; Senut et al., 1991; Su et al., 1997; Thal et al., 2000; Lace et al., 2009). Finally, DG/CA3 area represents a significant portion of the cross-sectional area of the hippocampus as a whole, and we may hide its contribution by controlling for hippocampal atrophy; nevertheless, it is important to recognize that the relationship between CA1-SRLM and ERC survived this same correction. We note that DG/CA3, which supplies a major projection to CA1-SRLM (Fig. 1), also did not correlate with CA1-SRLM thickness (Table 3). ERC width and hippocampal size together accounted for no more than $55 \%$ of the variance in CA1-SRLM width in our regression model (Table 4), suggesting that a significant portion of the variance may rely on other unmeasured structural or nonstructural factors.

CA1-SP and ERC shared a significant partial correlation (Table 3), but this relationship did not survive a regression analysis. This finding may reflect disproportionate vulnerability of the apical neuropil, rather than the CA1 pyramidal neuron cell bodies themselves, to AD pathology, a hypothesis supported by the postmortem neuropathology literature (Braak and Braak, 1997a; Thal et al., 2000; Scheff et al., 2007; Lace et al., 2009). Indeed, the clinical manifestations of $\mathrm{AD}$ probably reflect loss of synapses more than loss of the neurons themselves (Selkoe, 2002; Scheff et al., 2007).

Although the CA1-SRLM-to-ERC width ratio was stable on average across YCs and OCs and across a cognitive spectrum (Fig. 2 ), actual correlations between the two structures were not uniform between groups (see Results and Fig. 4). Indeed, there was no correlation among YCs, and the atrophy-corrected correlation among the older participants appeared to have been driven by OCs. Although statistical power may be the reason that a significant correlation was observed for this one group and not others (at $n=18$, OCs represented the largest group in our study), we speculate that structural covariance may be most evident between these two structures at an early, preclinical stage of pathology. Among our OCs, we have no way of determining the possible extent of asymptomatic neurofibrillary pathology, but incidental Braak stage 1-2 pathology is common in this age group (Braak and Braak, 1997b). It is in Braak stage 2 that neurofibrillary tau pathology first appears in the CA1-SRLM (ERC and perirhinal cortex are uniquely affected in stage 1 ), and by the time individuals reach stage 3 and beyond, tau pathology pervades the entire MTL (Braak and Braak, 1997a; Braak et al., 2006). This may explain why our partial correlations — which adjust for overall hippocampal atrophy-lost significance among patients with objective clinical findings (Fig. 4), who were more likely than OCs to harbor more pervasive pathology and generalized atrophy. Importantly, unadjusted Pearson's correlations between CA1SRLM and ERC widths held up across the diagnostic spectrum. Although speculative, the lack of a correlation in young controls may imply that structural covariance occurs only after the introduction of tau pathology. 
What accounts for structural covariance between the CA1SRLM and ERC? In the course of neurodegeneration, correlations in size presumably reflect proportional loss of tissue mass. The CA1-SRLM is composed mainly of dendrites, axons, and their interconnections, as well as sparse interneurons and support cells. The ERC contains these structures plus a large number of neuronal cell bodies. Our technique provides no information regarding which particular structures contribute to $\mathrm{AD}$-associated atrophy in these areas. Moreover, we cannot visualize any molecular pathology and can only speculate that tau aggregates are probably present in our patients. The factors that may explain structural covariance among healthy controls are unknown. The most simplistic explanation is that a greater number of projecting neurons in one structure (ERC) may correlate with a greater mass of axons and postsynaptic elements in the target structure (CA1-SRLM); however, the relationship may be more complex, with shared trophic factors or other influences. A reduction in the average dimensions of the ERC and CA1-SRLM in OCs versus YCs (Fig. 2) may reflect a nonspecific consequence of age or the presence of preclinical AD pathology.

MTL atrophy has important implications for episodic memory. Delayed free recall, a neuropsychological construct intended to isolate this core function of the hippocampus, correlates significantly with ERC, CA1-SRLM, and CA1-SP widths among patients with mild AD (Kerchner et al., 2012; also see Table 5). Among patients with aMCI, only CA1-SRLM bore a significant relationship with delayed recall, and OCs exhibited no significant MTL structural-behavioral correlate (Table 5). It may be the case that CA1-SRLM integrity is an early bottleneck to successful recall of recently learned stimuli as neurofibrillary pathology advances, and its atrophy may herald the onset of clinical symptoms. Of possible interest, the relationship between CA1-SRLM and delayed memory performance emerged at the same point in the diagnostic spectrum in which atrophyadjusted CA1-SRLM-to-ERC structural covariance lost significance (Fig. 4).

A limitation of our cross-sectional study is that it was not designed to determine the ordered succession of atrophy in the MTL. It would be of interest to pursue longitudinal imaging to test whether ERC thinning precedes that of the CA1-SRLM, as predicted from the postmortem pathology literature (Brady and Mufson, 1991; Braak and Braak, 1997a; Thal et al., 2000; Braak et al., 2006; Lace et al., 2009).

In summary, ERC and CA1-SRLM thinned from YCs to OCs and along an $\mathrm{AD}$ diagnostic spectrum, maintained a fixed ratio across all groups on average, and exhibited structural covariance in older subjects, particularly OCs. The same two structures are among the first in the MTL to demonstrate AD-related neurofibrillary tau pathology in postmortem specimens, and our in vivo imaging data support this notion of shared vulnerability.

\section{References}

Adachi M, Kawakatsu S, Hosoya T, Otani K, Honma T, Shibata A, Sugai Y (2003) Morphology of the inner structure of the hippocampal formation in Alzheimer disease. Am J Neuroradiol 24:1575-1581. Medline

Albert MS, DeKosky ST, Dickson D, Dubois B, Feldman HH, Fox NC, Gamst A, Holtzman DM, Jagust WJ, Petersen RC, Snyder PJ, Carrillo MC, Thies B, Phelps CH (2011) The diagnosis of mild cognitive impairment due to Alzheimer's disease: recommendations from the National Institute on Aging-Alzheimer's Association workgroups on diagnostic guidelines for Alzheimer's disease. Alzheimers Dement 7:270-279. CrossRef Medline
Apostolova LG, Dinov ID, Dutton RA, Hayashi KM, Toga AW, Cummings JL, Thompson PM (2006) 3D comparison of hippocampal atrophy in amnestic mild cognitive impairment and Alzheimer's disease. Brain 129: 2867-2873. CrossRef Medline

Braak E, Braak H (1997a) Alzheimer's disease: transiently developing dendritic changes in pyramidal cells of sector CA1 of the Ammon's horn. Acta Neuropathol 93:323-325. CrossRef Medline

Braak H, Braak E (1997b) Frequency of stages of Alzheimer-related lesions in different age categories. Neurobiol Aging 18:351-357. CrossRef Medline

Braak H, Alafuzoff I, Arzberger T, Kretzschmar H, Del Tredici K (2006) Staging of Alzheimer disease-associated neurofibrillary pathology using paraffin sections and immunocytochemistry. Acta Neuropathol 112:389404. CrossRef Medline

Brady DR, Mufson EJ (1991) Alz-50 immunoreactive neuropil differentiates hippocampal complex subfields in Alzheimer's disease. J Comp Neurol 305:489-507. CrossRef Medline

Csernansky JG, Wang L, Swank J, Miller JP, Gado M, McKeel D, Miller MI, Morris JC (2005) Preclinical detection of Alzheimer's disease: hippocampal shape and volume predict dementia onset in the elderly. Neuroimage 25:783-792. CrossRef Medline

Cummings JL, Mega M, Gray K, Rosenberg-Thompson S, Carusi DA, Gornbein J (1994) The Neuropsychiatric Inventory: comprehensive assessment of psychopathology in dementia. Neurology 44:2308-2314. CrossRef Medline

de Calignon A, Polydoro M, Suárez-Calvet M, William C, Adamowicz DH, Kopeikina KJ, Pitstick R, Sahara N, Ashe KH, Carlson GA, Spires-Jones TL, Hyman BT (2012) Propagation of tau pathology in a model of early Alzheimer's disease. Neuron 73:685-697. CrossRef Medline

Frost B, Jacks RL, Diamond MI (2009) Propagation of Tau misfolding from the outside to the inside of a cell. J Biol Chem 284:12845-12852. CrossRef Medline

Harris JA, Koyama A, Maeda S, Ho K, Devidze N, Dubal DB, Yu GQ, Masliah E, Mucke L (2012) Human P301L-mutant tau expression in mouse entorhinal-hippocampal network causes tau aggregation and presynaptic pathology but no cognitive deficits. PLoS One 7:e45881. CrossRef Medline

Insausti R, Amaral DG (2012) Hippocampal formation. In: Atlas of the human brain, Ed 3 (Mai JK, Paxinos G, eds), pp 896-942. London: Elsevier Academic.

Kerchner GA (2011) Ultra-high field 7T MRI: a new tool for studying Alzheimer's disease. J Alzheimer's Dis 22 [Supp 3]:91-95. CrossRef

Kerchner GA, Hess CP, Hammond-Rosenbluth KE, Xu D, Rabinovici GD, Kelley DA, Vigneron DB, Nelson SJ, Miller BL (2010) Hippocampal CA1 apical neuropil atrophy in mild Alzheimer disease visualized with 7-T MRI. Neurology 75:1381-1387. CrossRef Medline

Kerchner GA, Deutsch GK, Zeineh M, Dougherty RF, Saranathan M, Rutt BK (2012) Hippocampal CA1 apical neuropil atrophy and memory performance in Alzheimer's disease. Neuroimage 63:194-202. CrossRef Medline

Killiany RJ, Hyman BT, Gomez-Isla T, Moss MB, Kikinis R, Jolesz F, Tanzi R, Jones K, Albert MS (2002) MRI measures of entorhinal cortex vs hippocampus in preclinical AD. Neurology 58:1188-1196. CrossRef Medline

Lace G, Savva GM, Forster G, de Silva R, Brayne C, Matthews FE, Barclay JJ, Dakin L, Ince PG, Wharton SB; MRC-CFAS (2009) Hippocampal tau pathology is related to neuroanatomical connections: an ageing population-based study. Brain 132:1324-1334. CrossRef Medline

Liu L, Drouet V, Wu JW, Witter MP, Small SA, Clelland C, Duff K (2012) Trans-synaptic spread of tau pathology in vivo. PLoS One 7:e31302. CrossRef Medline

McKhann G, Drachman D, Folstein M, Katzman R, Price D, Stadlan EM (2011) Clinical diagnosis of Alzheimer's disease: Report of the NINCDSADRDA Work Group under the auspices of Department of Health and Human Services Task Force on Alzheimer's Disease. Neurology 77:333. CrossRef Medline

Meng XL, Rosenthal R, Rubin DB (1992) Comparing correlated correlation coefficients. Psychol Bull 111:172-175. CrossRef

Morris JC (1993) The Clinical Dementia Rating (CDR): current version and scoring rules. Neurology 43:2412-2414. CrossRef Medline

Mueller SG, Stables L, Du AT, Schuff N, Truran D, Cashdollar N, Weiner MW (2007) Measurement of hippocampal subfields and age-related changes 
with high resolution MRI at 4T. Neurobiol Aging 28:719-726. CrossRef Medline

Mueller SG, Schuff N, Yaffe K, Madison C, Miller B, Weiner MW (2010) Hippocampal atrophy patterns in mild cognitive impairment and Alzheimer's disease. Hum Brain Mapp 31:1339-1347. CrossRef Medline

Pfeffer RI, Kurosaki TT, Harrah CH Jr, Chance JM, Filos S (1982) Measurement of functional activities in older adults in the community. J Gerontol 37:323-329. CrossRef Medline

Rosset A, Spadola L, Ratib O (2004) OsiriX: an open-source software for navigating in multidimensional DICOM images. J Digit Imaging 17:205216. CrossRef Medline

Scheff SW, Price DA, Schmitt FA, DeKosky ST, Mufson EJ (2007) Synaptic alterations in CA1 in mild Alzheimer disease and mild cognitive impairment. Neurology 68:1501-1508. CrossRef Medline

Seeley WW, Crawford RK, Zhou J, Miller BL, Greicius MD (2009) Neurodegenerative diseases target large-scale human brain networks. Neuron 62:42-52. CrossRef Medline

Selkoe DJ (2002) Alzheimer's disease is a synaptic failure. Science 298:789791. CrossRef Medline

Senut MC, Roudier M, Davous P, Fallet-Bianco C, Lamour Y (1991) Senile dementia of the Alzheimer type: is there a correlation between entorhinal cortex and dentate gyrus lesions? Acta Neuropathol 82:306-315. CrossRef Medline

Su JH, Deng G, Cotman CW (1997) Transneuronal degeneration in the spread of Alzheimer's disease pathology: immunohistochemical evidence for the transmission of tau hyperphosphorylation. Neurobiol Dis 4:365375. CrossRef Medline

Thal DR, Holzer M, Rüb U, Waldmann G, Günzel S, Zedlick D, Schober R (2000) Alzheimer-related tau-pathology in the perforant path target zone and in the hippocampal stratum oriens and radiatum correlates with onset and degree of dementia. Exp Neurol 163:98-110. CrossRef Medline

Xu Y, Jack CR Jr, O'Brien PC, Kokmen E, Smith GE, Ivnik RJ, Boeve BF, Tangalos RG, Petersen RC (2000) Usefulness of MRI measures of entorhinal cortex versus hippocampus in AD. Neurology 54:1760-1767. CrossRef Medline

Yassa MA, Stark SM, Bakker A, Albert MS, Gallagher M, Stark CE (2010) High-resolution structural and functional MRI of hippocampal CA3 and dentate gyrus in patients with amnestic mild cognitive impairment. Neuroimage 51:1242-1252. CrossRef Medline

Yesavage JA (1988) Geriatric depression scale. Psychopharmacol Bull 24: 709-711. Medline 